\title{
On the probability of solar cosmic ray fluency during SEP event in dependence of the level of solar activity
}

\author{
L.I. Dorman ${ }^{\mathrm{a}, \mathrm{b}}$ and L.A. Pustil'nik ${ }^{\mathrm{a}}$ \\ (a) Israel Cosmic Ray Space Weather Center and Emilio Segre' Observatory, Tel Aviv University,69978, ISRAEL; \\ (b) Cosmic Ray Department of IZMIRAN Russian Academy of Science, RUSSIA; \\ Presenter: L. Pustil'nik (levpust@post.tau.ac.il), isr-pustilnik-L-abs2-sh35-poster
}

We estimate probabilities of radiation hazard (fluency) in the Earth environment for space probes, satellites and astronauts depend on phase of solar activity and energy of CR protons. These probabilities we calculate on the basis of available data on ground and satellite direct measurements of solar CR fluencies for the last several solar cycles, and especially satellite data for 1994-2004. This research is in the frame of INTAS 810 and new Project COST 724.

\section{Introduction}

In papers Dorman et al. (1993), Dorman and Pustil'nik (1995, 1999) were found the frequencies P (event/year) of solar CR fluencies F (particles/cm2) in dependence of fluency value F averaged for solar cycle and for sunspot number intervals. Here, on the basis of data from satellites GOES (GOES-7 for 1994, GOES-8 for 1995-1996 and 1999-2002, GOES-9 for 1997-1998, and GOES-11 for 2003-2004), (http://www.sec.noaa.gov/ftpmenu/indices/old indices.html) with 11-year data on the fluencies of particles with energies $>1 \mathrm{MeV},>10 \mathrm{MeV}$, and $>100 \mathrm{MeV}$ for the period 1994-2004 we analyze statistical properties of fluency in different states of solar activity and for particles with different energies.

Main answers of our analysis are:

1. What kind of CR activity is most dangerous - long time accumulation of the low intensity backgrouns or short time exposition of particles from strong solar flares?

2. What probability of the given fluency for different energies of the particles and does it depend on soalr activity or some universal parameter.

\section{Summarize of our previous results; approximation by analytical formulas}

In Fig. 1a and Fig $1 \mathrm{~b}$ are shown the dependencies of events frequency $\mathrm{P}$ (events/year) from the value of logarithm of fluency $\lg (\mathrm{F})$ of solar protons with energy $>10 \mathrm{MeV}$ and $>30 \mathrm{Mev}$ for different levels of solar activity: $\mathrm{W}=\tilde{0} 40,40-80,80-120,120-160,160-200$.
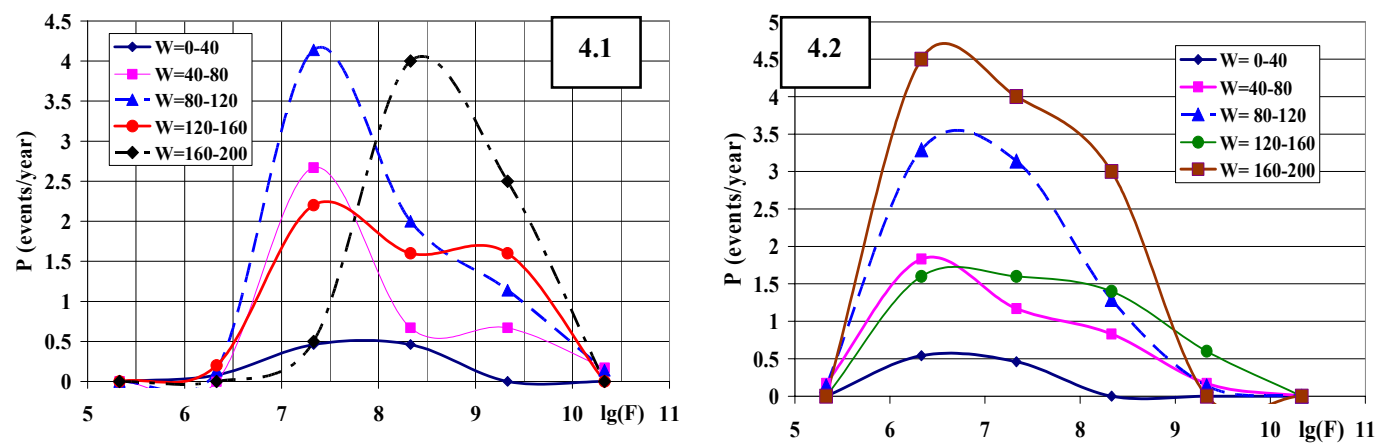

Fig 1a: Events frequency P(lgF) for $>10 \mathrm{MeV}$ CR for different Fig.1b: the same for more high energy CR $(>30 \mathrm{MeV})$. sunspot activity W.

It can be seen from Fig. 1, that the number of events with high fluency depends strongly from both: sunspot solar activity and energy of the particles.

3. Statistical properties of the radiation hazard frequencies delivered from goes $\tilde{7 / 11}$ for 1994-2004 data on daily fluencies 
We analyzed 11-year data on daily fluencies (number of particles with energy more then $1 \mathrm{MeV}, 10$ $\mathrm{MeV}$, and $100 \mathrm{MeV}$, accumulated by $1 \mathrm{~cm}^{2}$ from 1 steradian during one day with energies $1 \mathrm{MeV}, 10 \mathrm{MeV}$, and $100 \mathrm{MeV}$ ) from satellites GOES (GOES-7 for 1994, GOES-8 for 1995-1996 and 1999-2002, GOES-9 for 1997-1998, and GOES-11 for 2003-2004), available as Daily Particle Indices from Internet (http://www.sec.noaa.gov/ftpmenu/indices/old_indices.html) (see Fig. 6). As it may be seen from the Fig. 2, daily fluencies include 2 components: background and SEP-Solar Energetic Particles. Backgrounds for different energies changes from daily fluency about $10^{3.5}$ particle.cm ${ }^{-2}$ for energies $>100 \mathrm{MeV}$, about $10^{4}$ particle. $\mathrm{cm}^{-2}$ for energies $>10 \mathrm{MeV}$ and from $10^{5}$ up to $10^{6.5}$ particle.cm ${ }^{-2}$ for energy $>1 \mathrm{MeV}$. High variatiability of the low energy background caused probably by the Earth's radiation belts input.

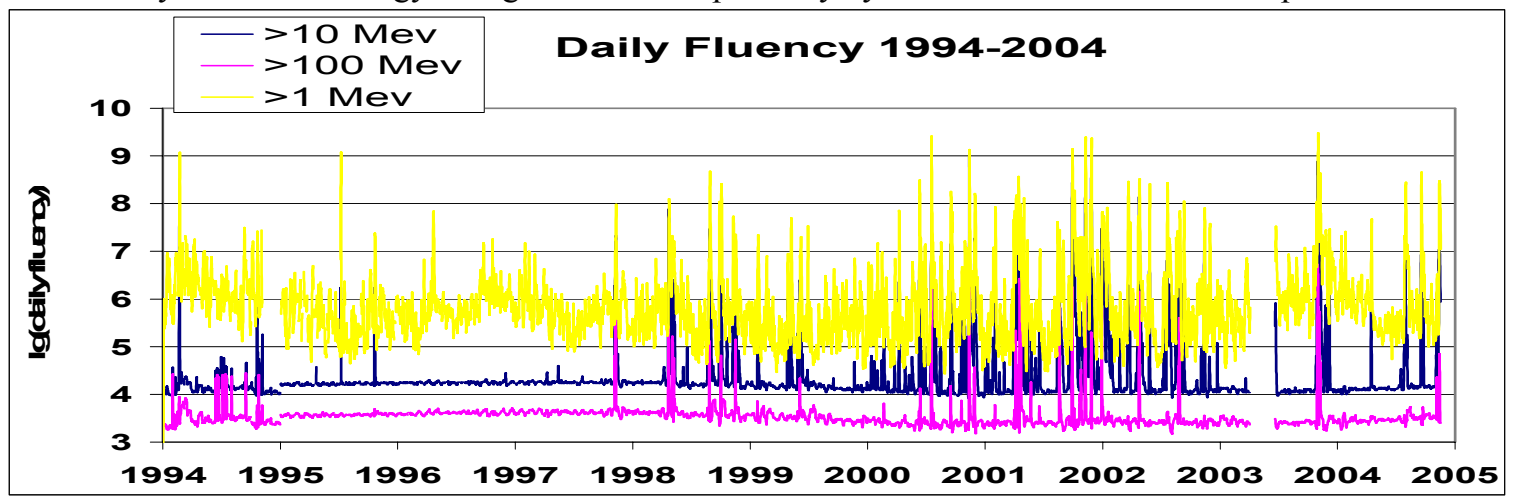

Fig.2. Data of GOES satellites of daily CR fluency.

Integrated solar proton fluencies accumulated during total 11- year period 1994-2004 for each different interval of fluencies are shown in Fig. 3. From Fig. 3 can be seen that integrated input to accumulated proton fluency during total 1994-2004 period from rare but strong events (daily fluency $>10^{9}$ particle/( $\mathrm{cm}^{2}$ ster $)$ ) is much more then input from numerous weak events (noise like) with daily fluencies $10^{4.5}-10^{7}$ particle/ $\left(\mathrm{cm}^{2}\right.$ ster $)$. It is correct both for fluencies, included low energy particles $(\mathrm{E}>1 \mathrm{MeV})$ and for fluencies of high energy particles with energy $>10 \mathrm{MeV}$ and $>100 \mathrm{MeV}$. Another conclusion from Fig. 3 can be made about frequency-fluency spectrum: dependence of frequency - number of days $\mathrm{N}(\mathrm{F})$ with given daily fluency $F$ has power character $\mathrm{N}(\mathrm{F})=\mathrm{No}\left(\mathrm{F} / \mathrm{F}_{\mathrm{o}}\right)^{-\mathbf{k}}$.

The exponent value $\mathrm{k}$ changed from $\mathrm{k}=0.6$ for particles with energy $>1 \mathrm{MeV}, \mathrm{k}=0.4$ for particles with energy $>10 \mathrm{MeV}$ and $\mathrm{k}=0.2$ for particles with energy $>100 \mathrm{MeV}$.
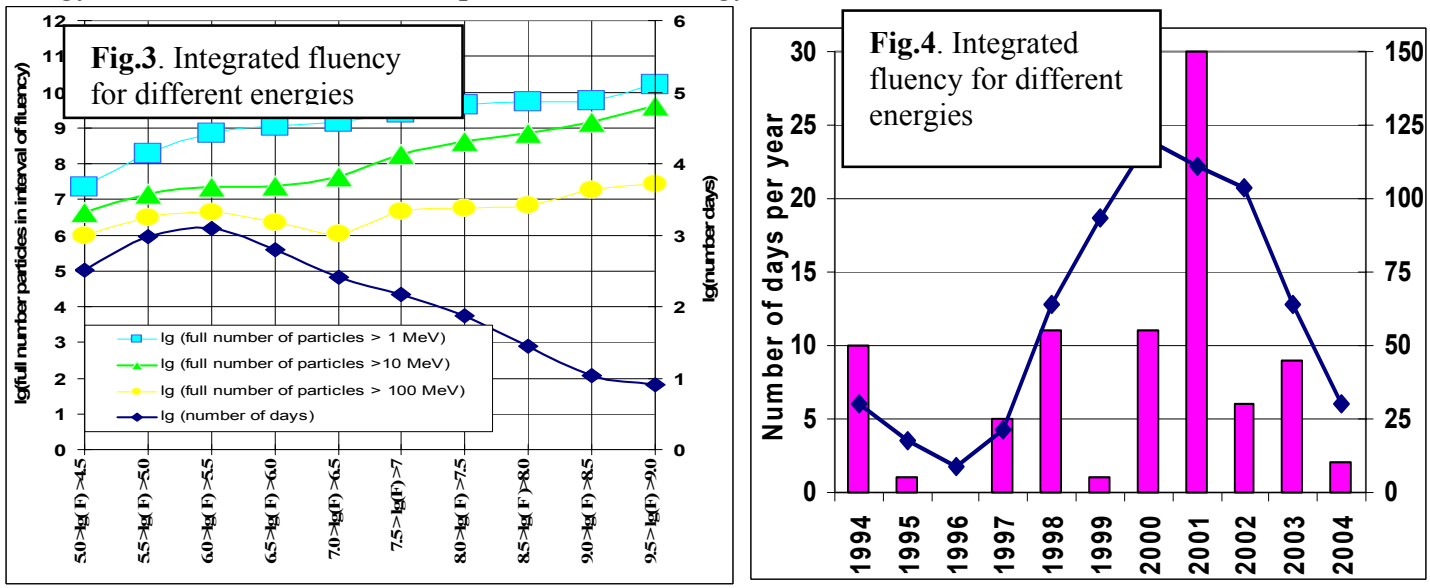

In Fig 4 are shown variations of sunspot number and number of days $\mathrm{N}$ with daily fluencies $\mathrm{F}(\mathrm{E}>100 \mathrm{MeV})$ $>10^{4}$ particle/(cm2 ster) during 1994 - 2004. From Fig. 4 can be seen that number of days with high fluency events is in generally in some evident correlation with level of sunspot activity. However, this connection is 
not so strong (correlation coefficient only 0.55); it includes big variances from proportional law. For examples let us consider 1999 year (1 year before maximum of solar activity) when strong SEP fluencies almost was absent. It means that sunspot number as unique parameter is not adequate for control and forecasting of great SEP fluency levels: there is quite large probability to obtain high fluency event near minimum phase of sunspot number, and correspondently, very low fluency input near maximum state of solar activity.

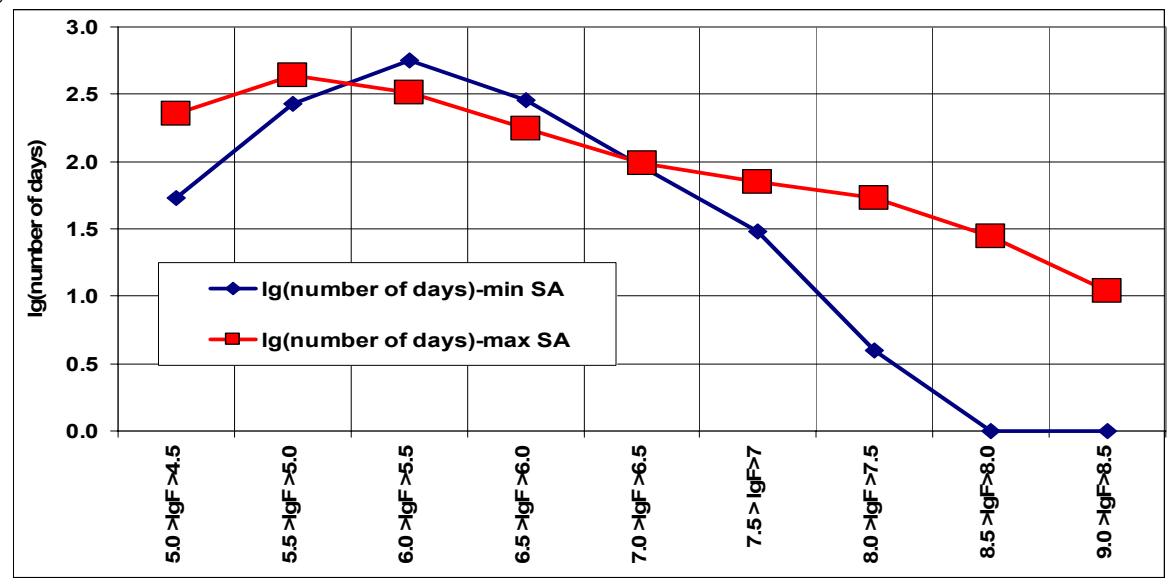

Fig. 5. Min/Max Asymmetry of fluencies distribution

In Fig. 5 we show the asymmetry of high fluency SEP for high energy particles $(\mathrm{E}>100 \mathrm{MeV})$ relative to the maximum and minimum states of solar activity. We see that this asymmetry exist in really but its level decreases with decreasing of the level of fluency: for $\mathrm{F}(\mathrm{E}>100 \mathrm{MeV})>10^{7}$ particle $/\left(\mathrm{cm}^{2}\right.$ ster $)$ we see only 2 events in 2000 and 1 in 2003 (near maximum of solar activity), and nothing near minimum of solar activity; for $\mathrm{F}(\mathrm{E}>100 \mathrm{MeV})>10^{6}$ particle/( $\mathrm{cm}^{2}$ ster) we see 3 events in $2000-2001$ (near maximum of solar activity), 2 events in 2002 - 2003 (the fall of solar activity), but also 1 event in 1997 (near minimum of solar activity). With decreasing of the level of $\mathrm{F}(\mathrm{E}>100 \mathrm{MeV})$ to $>10^{5}$ particle $/(\mathrm{cm} 2 \mathrm{ster})$ and $>10^{4}$ particle $/\left(\mathrm{cm}^{2}\right.$ ster) the asymmetry decreases essentially.

Another manifestation of this asymmetry we see in the Fig. 5, which shows the difference in frequency-fluency distributions of SEP for minimal and maximal states of solar activity. As it is can be seen from Fig. 5, this asymmetry in the low energy range is not trivial: for low fluencies days near the background level from $\lg (\mathrm{F}(\mathrm{E}>1 \mathrm{MeV}))=5$ up to value 7.5 different between maximum state of the solar activity and the minimum one is negligible or even opposite to expected from standard approach. Namely, fluency days with $\lg (\mathrm{F}(\mathrm{E}>1 \mathrm{MeV}))$ for fluency levels with $5<\lg (\mathrm{F}(\mathrm{E}>1 \mathrm{MeV}))<7$ is in minimal state of solar activity even more then in the maximum one. Only for high fluency events with $\lg (\mathrm{F}(\mathrm{E}>1 \mathrm{MeV}))>7$ we observe evident dominating of SEPs in maximal solar activity state. Possible reason of the absence or opposite attraction of low fluency events to solar maximum may be caused by input to low fluency state on the low energies of Earth radiation belts, what are in some anticorrelation with sunspot activity.

\section{On connection between SEP fluencies for different channels of particle energy; two different populations of particle fluencies}

Next question is possible connection between FEP Fluencies for different channel of particles energy. In another words - is fluency of high energy particles $(>100 \mathrm{MeV})$ depends on the level of fluency in low $(>1$ $\mathrm{MeV})$ or medium $(>10 \mathrm{MeV})$ energy channels? For this aim we constructed two diagrams: a) $\lg (\mathrm{F}(>10$ $\mathrm{MeV},>100 \mathrm{MeV})$ ) from $\lg \mathrm{F}(>1 \mathrm{MeV})-$ Fig. 6 , and b) $\lg \mathrm{F}(>100 \mathrm{MeV})$ from $\lg \mathrm{F}(>10 \mathrm{MeV})-$ Fig. 7

From Fig. 6 and Fig. 7 we observed input in the observed fluencies from two different populations of events with different source of the particles: first group provide input to low energy particles with wide interval of 
fluency $(\lg (\mathrm{F}(>1 \mathrm{MeV})=4.5-8.5)$ and does not give any input to medium and high energy fluency. Possible source of this particle is radiation belts of the Earth magnetosphere.

Second group of the SEP has higher amplitude of SEP fluencies and show evident correlation between fluencies in high energy and medium energy (and between high energy and low energy) particles input to fluency effect.
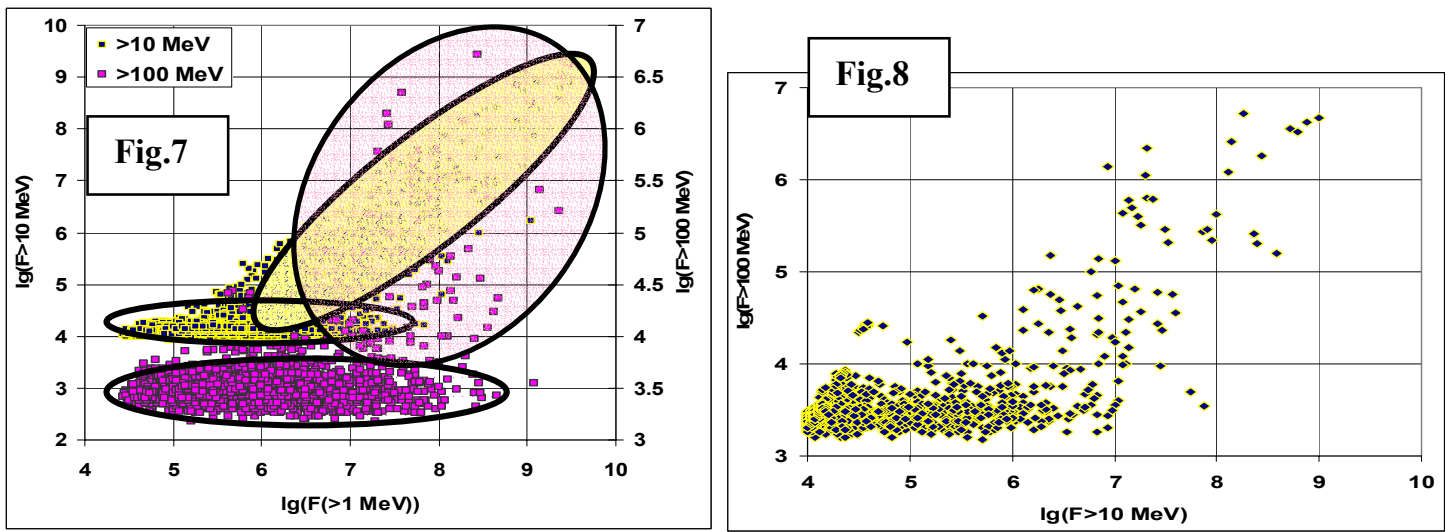

Fig.6. Two populations fluencies in 1-10, 1-100 Mev. Fig.7. Two populations fluencies in 10-100 Mev.

\section{Conclusions}

1. On the basis of 11 year observations on GOES 7-11 for 1994-2004 of daily fluencies was shown that only several rare events with the highest fluencies gave the main input to the integral fluency during 1994-2004.

2. Fluencies for different energy channels demonstrate different asymmetry relative to minimum and maximum states of solar activity.

2. Energy spectrum of the observed fluencies and its distribution in 2-D diagrams (Fluency(low energies)Fluency(high energies)) show existing of the two different populations of events: first population contents events in low energy particles without any reaction in high energy particles fluencies; second population contents very strong (caused by solar flares) events with simultaneous about proportional reaction in all energetic channels from $>1 \mathrm{MeV}$ to $>100 \mathrm{MeV}$ (regression coefficients about 0.9 and correlation coefficients 0.7-0.8).

4. Our preliminary estimation of the radiation doses in space from the greatest SEP events with fluencies $F(E$ $>1 \mathrm{MeV})>10^{9}$ particle $/\left(\mathrm{cm}^{2}\right.$ ster $)$ show that this group of events is source of real radiation hazard (tens hundreds rem during event).

\section{Acknowledgements}

Our thanks to GOES team presented the observation data for 1994 - 2004 to open excess. Our thanks also to Prof. Yuval Ne'eman, Dr. Abraham Sternlieb, Dr. Zvi Kaplan, and Dr. Gideon Bella for useful discussions and permanent support. This research was partly supported by COST -724 .

\section{References}

[1] L.I. Dorman, Cosmic Rays in the Earth's Atmosphere and Underground, Kluwer Ac. Publ., Dordrecht/Boston/London, 2004.

[2] I.V. Dorman., L.I. Dorman and D. Venkatesan,. Proc. 23-th Intern. Cosmic Ray Conf., Calgary, 3, 79$82,1993$.

[3] L.I. Dorman and L.A. Pustil'nik, Proc. 24-th Intern. Cosmic Ray Conf., Rome, 4, 86-89, 1995.

[4] L.I. Dorman and L.A. Pustil'nik, Proc. 26-th Intern. Cosmic Ray Conf., Salt Lake City, 6, 407-410, 1999.

[5] K.G. McCracken, D.F. Smart, M.A. Shea, and G.A.M. Dreschhoff, Proc. 27-th Intern. Cosmic Ray Conf., Hamburg, 8, 3209-3212, 2001.

[6] M.A. Shea and D.F. Smart, Solar Physics, 127, 297-320, 1990. 